\title{
AL 3 (BH 261): A NEW GLOBULAR CLUSTER IN THE GALAXY ${ }^{1}$
}

\author{
S. Ortolani, ${ }^{2,3}$ E. Bica, ${ }^{4}$ AND B. BarbUY ${ }^{5}$ \\ Received 2006 June 9; accepted 2006 June 22; published 2006 July 20
}

\begin{abstract}
AL 3 (BH 261), previously classified as a faint open cluster candidate, is shown to be a new globular cluster in the Milky Way, by means of $B, V$, and $I$ color-magnitude diagrams. The main feature of AL 3 is a prominent blue extended horizontal branch. Its color-magnitude diagrams match those of the intermediate-metallicity cluster M5. The cluster is projected in a rich bulge field, also contaminated by the disk main sequence. The globular cluster is located in the Galactic bulge at a distance from the Sun $d_{\odot}=6.0 \pm 0.5 \mathrm{kpc}$. The reddening is $E(B-V)=0.36 \pm 0.03$, and the metallicity is estimated to be $[\mathrm{Fe} / \mathrm{H}] \approx-1.3 \pm 0.25$. AL 3 is probably one of the least massive globular clusters of the Galaxy.
\end{abstract}

Subject headings: globular clusters: individual (AL 3) - Hertzsprung-Russell diagram

\section{INTRODUCTION}

New globular clusters have been discovered or identified in the Galaxy in the last few years. Let us recall Lyngå 7 (Ortolani et al. 1993; Tavarez \& Friel 1995), Pyxis (Weinberger 1995; Da Costa 1995; Irwin et al. 1995; Sarajedini \& Geisler 1996), and IC 1257 (Harris et al. 1997). The Two Micron All Sky Survey (2MASS) has provided us with the two new globulars 2MASS-GC01 and 2MASS-GC02 (Hurt et al. 2000). Ortolani et al. (2000) identified ESO 280-SC06 as a halo globular cluster projected on the disk that was previously classified as an open cluster. Kobulnicky et al. (2005) discovered GLIMPSE-C01 from an inspection of Spitzer Space Telescope images. Carraro (2005) identified Whiting 1 (Whiting et al. 2002) as a young globular cluster in the halo. Finally, Willman et al. (2005) reported the discovery of SDSS J1049+5103, which appears to be a globular cluster or a dwarf galaxy in the outer halo.

The Harris (1996) catalog of globular clusters, ${ }^{6}$ as updated in 2003 February, contains 150 objects. Adding the three new objects from 2005, the number of Galactic globular clusters amounts to 153 .

AL 3 was discovered by Andrews \& Lindsay (1967) and was also cataloged as BH 261 by van den Bergh \& Hagen (1975). It also appears in the ESO/Uppsala catalog (Lauberts 1982) as ESO 456-SC78. Its angular size was estimated to be $\sim 1$ '.5. The cluster was recently observed by Carraro et al. (2005) using CCD images of $4.1 \times 4{ }^{\prime} .1$, centered on the object, but they concluded from color-magnitude diagrams (CMDs) that no cluster was present.

In the present study, we show AL 3 to be the 154th globular cluster in the Galaxy. We measured the cluster center by means of DSS/XDSS images and found that it is located at $\alpha=$ $18^{\mathrm{h}} 14^{\mathrm{m}} 06^{\mathrm{s}} 6, \delta=-28^{\circ} 38^{\prime} 06^{\prime \prime}(\mathrm{J} 2000)$, with Galactic coordinates $l=3.36, b=-5^{\circ} .27$. In $\S 2$, we describe the observations and reductions. In $\S 3$, we present CMDs and derive the parameters. Concluding remarks are given in $\S 4$.

\footnotetext{
${ }^{1}$ The observations were carried out at the European Southern Observatory, La Silla, Chile [proposal 64L-0212(A)].

${ }^{2}$ Università di Padova, Dipartimento di Astronomia, Vicolo dell'Osservatorio 5, I-35122 Padova, Italy; ortolani@pd.astro.it.

${ }^{3}$ Visiting Astronomer, European Southern Observatory, La Silla, Chile.

${ }^{4}$ Universidade Federal do Rio Grande do Sul, Departamento de Astronomia, CP 15051, Porto Alegre 91501-970, Brazil; bica@if.ufrgs.br.

${ }^{5}$ Universidade de São Paulo, Departamento de Astronomia, Rua do Matão 1226, São Paulo 05508-900, Brazil; barbuy@astro.iag.usp.br.

${ }^{6}$ See http://www.physics.mcmaster.ca/Globular.html.
}

\section{OBSERVATIONS}

AL 3 was observed on 2000 March 6 with the $1.54 \mathrm{~m}$ Danish telescope at ESO (La Silla). A Loral/Lesser CCD detector C1W7 with $2052 \times 2052$ pixels, of pixel size $15 \mu \mathrm{m}$, was used. A pixel corresponds to 0.39 on the sky, and the full field of the camera is $13^{\prime} \times 13^{\prime}$. Images in $B$ ( 3 minutes), $V$ ( 1 and 3 minutes $)$, and $I$ (10 and $40 \mathrm{~s})$ were obtained, under a seeing of 1".2. In Figure 1 , we show a 3 minute $B$ exposure of AL 3 for a field extraction of $3.3 \times 3.3(510 \times 510$ pixels $)$. The image suggests a small concentration of stars $\left(24^{\prime \prime} \times 12^{\prime \prime}\right)$ surrounded by a halo of fainter stars. The overall appearance is similar to that of the bulge globular cluster NGC 6540 (Bica et al. 1994), but somewhat fainter.

DAOPHOT II (Stetson 1987) was used to extract the instrumental magnitudes. For calibrations, we used Landolt (1983, 1992) standard stars. The calibration equations are $V=$ $26.46+0.01(B-V)+v$ for $10 \mathrm{~s}, B=26.40+0.1(B-V)$ $+b$ for $10 \mathrm{~s}$, and $I=24.61-0.01(V-I)+i$ for $15 \mathrm{~s}$, at 1.25 air masses. Zero-point errors are dominated by the transfer from aperture to DAOPHOT PSF-fitting magnitudes and CCD shutter time uncertainties. Atmospheric extinction errors are smaller since AL 3 and standard stars were observed at comparable air masses. Transfer errors arise from noise in the stellar profile growth curve due to crowding ( $\approx 1$ star per 70 pixels). This contaminates the stellar wings even for the brightest and relatively isolated stars. We investigate three methods: (1) statistical reconstruction of the growth curve in small radius bins for the best $\approx 100$ stars per band; (2) analysis of a few relatively isolated bright stars by subtracting contaminating stars (CSs) with the point-spread function (PSF); and (3) growth curve analysis using models (Diego 1985). The first method gives the smallest errors (0.02-0.03 mag), while in the second method, subtraction residuals increase noise in the star wings. This is caused by possible underestimates of the sky around the CSs or could be due to PSF deviations for faint stars and steeper profiles. The second method should be free of statistical errors and agrees with the third method within a few hundredths of a magnitude for Lorentzian profiles. Gaussian profiles show $\approx 5$ mag. The CCD shutter time uncertainties $(0.3 \mathrm{~s})$ related to short exposures done for standard stars lead to an additional 3\% error, which is propagated to the calibrations of the long-exposure cluster frames. The final magnitude zero-point uncertainty is estimated to be $\pm 0.05 \mathrm{mag}$. The atmospheric extinction was corrected with the La Silla standard coefficients. 


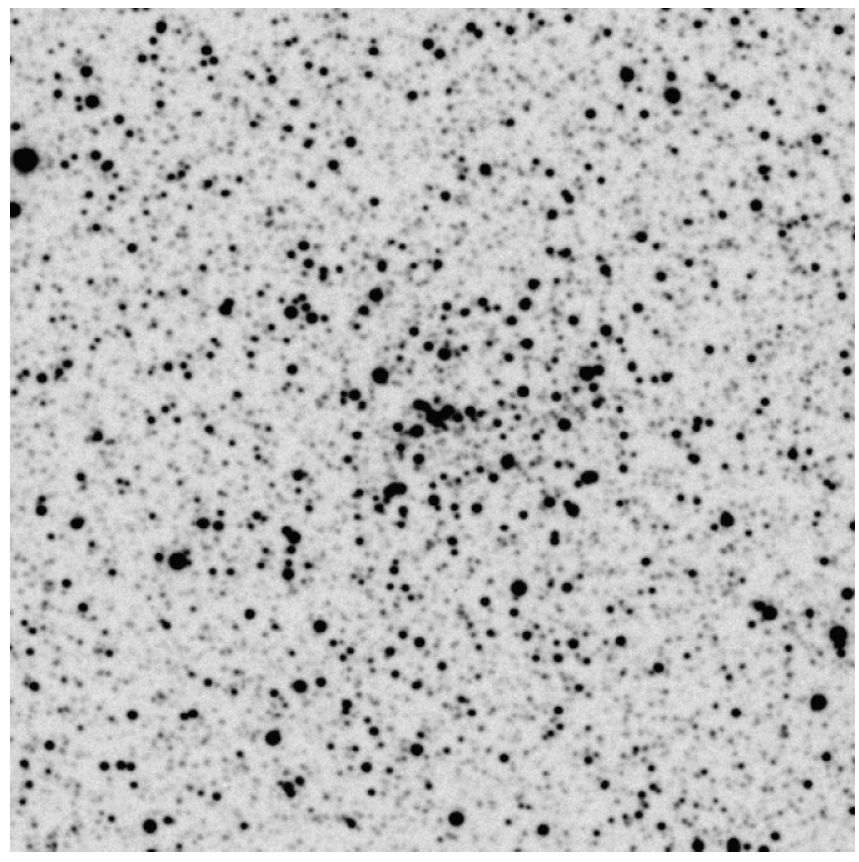

FIG. 1.-A 3 minute $B$ image of AL 3 for a field extraction of $3^{\prime} \times 3^{\prime}$. North is up, and east is left.

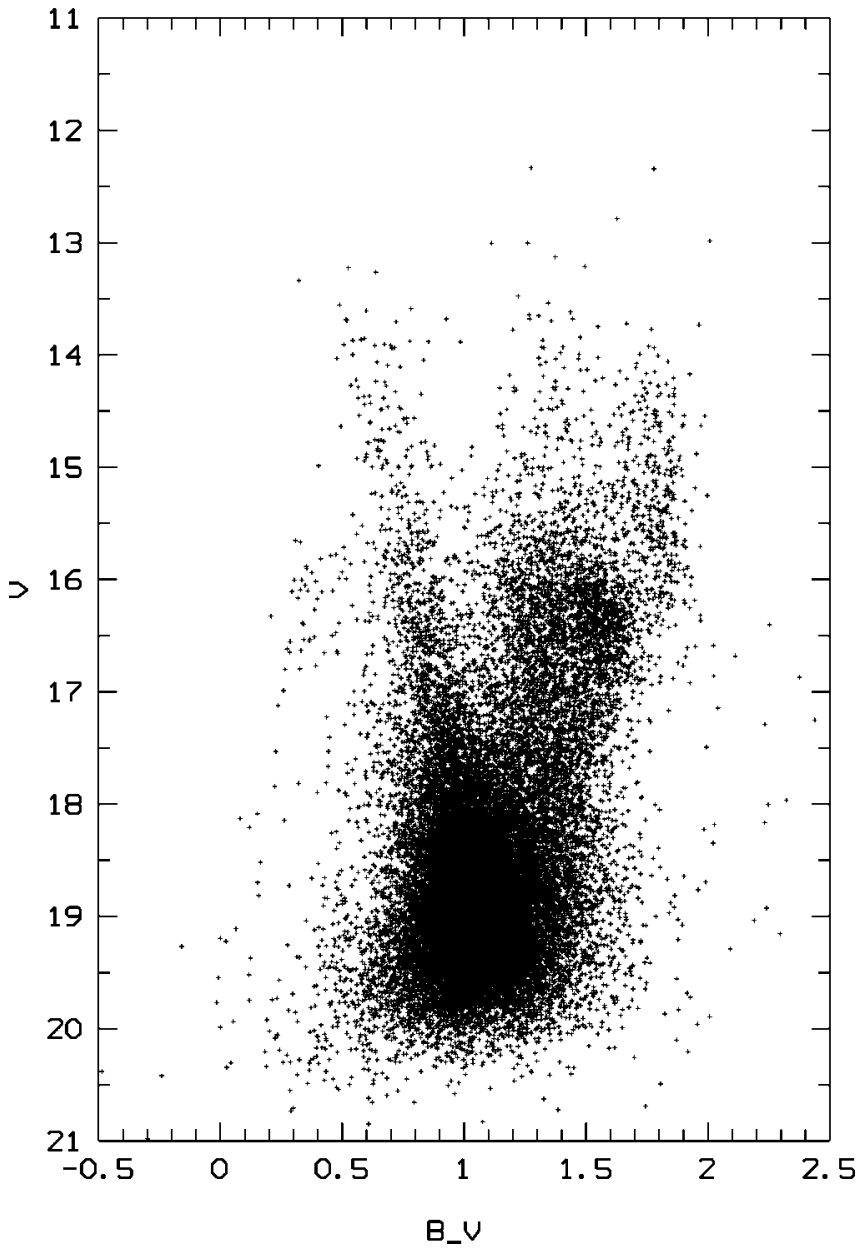

Fig. 2. $-V$ vs. $B-V$ for the whole field $\left(13^{\prime} \times 13^{\prime}\right)$.

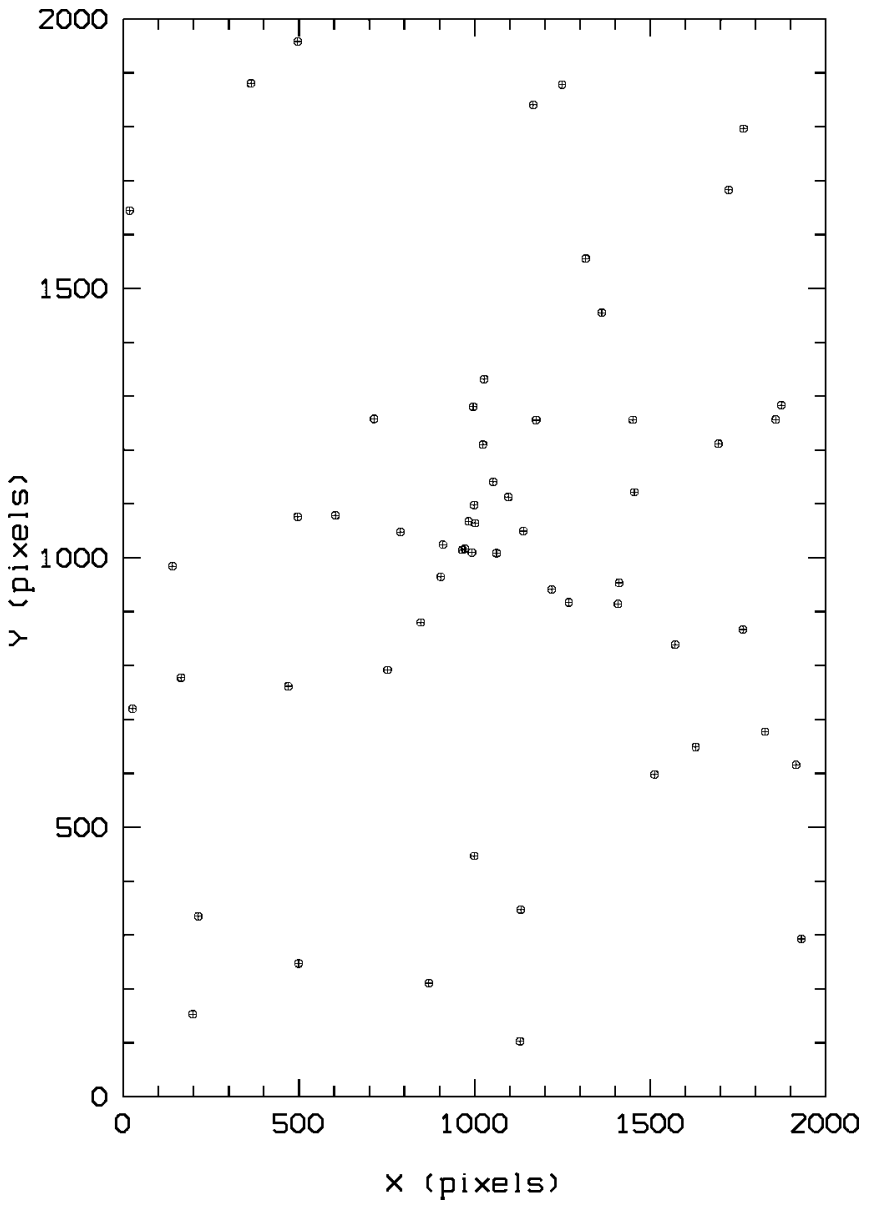

FIG. 3.-Whole field $\left(13^{\prime} \times 13^{\prime}\right)$ in $(X, Y)$-pixels showing the distribution of blue $\mathrm{HB}$ stars, defined as $15.5<V<17.1$ and $0<B-V<0.55$.

\section{COLOR MAGNITUDE DIAGRAMS}

Figure 2 shows the $V$ versus $B-V$ CMD of the full field. It is a typical bulge CMD similar to that of Baade's window (e.g., Terndrup 1988). The prominent features are a bulge red horizontal branch (HB) at $V \approx 16.5, B-V \approx 1.5$, a metal-rich red giant branch (RGB) with a turnover such as in NGC 6553 (Ortolani et al. 1990), a disk blue main sequence, and a blue extended $\mathrm{HB}$ with an overdensity at $V=16, B-V=0.4$. In Figure 3, we show the spatial distribution across the whole field of the stars in a box encompassing the blue HB (BHB) in the CMD. The BHB stars are centrally concentrated, revealing a globular cluster. We study the stellar density profile derived from the BHB stars of Figure 3, which are relatively free of contamination. The limiting radius (where the density profile merges with the background) is $r_{\lim }=3.4 \pm 0.4$. The tidal radius must be larger since some BHB stars reach the field borders (Fig. 3). Low statistics precludes a King profile fit, and deeper images using field decontaminated turnoff (TO) and main-sequence stars would be necessary to explore the possibility of post-core collapse. From the observed density profile, we estimate a half-density radius $r_{\text {hd }}=1^{\prime}-1.5$.

Figure 4 shows a $V$ versus $B-V$ CMD for a cluster extraction of $r<120$ pixels $\left(r<47^{\prime \prime}\right)$ and a more central one with $r<53$ pixels $\left(r<21^{\prime \prime}\right)$ overlaid, of the same order of the core seen in Figure 1. A few giants are present, which is typical of Palomar clusters, and the TO seems to be reached near the photometry limit. Coordinates of the giants are available by request. As comparison the mean locus of M5 $([\mathrm{Fe} / \mathrm{H}]=-1.27$; Harris 


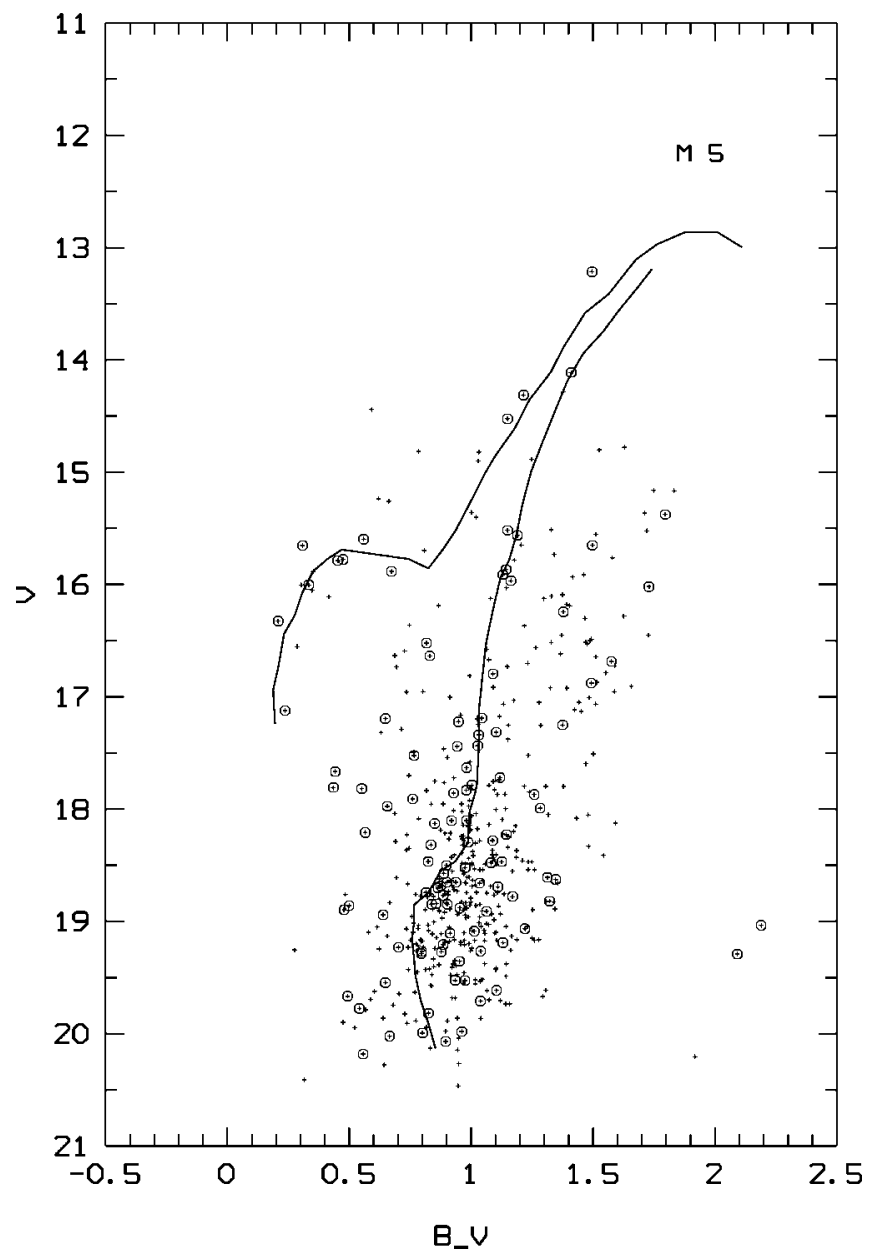

Fig. 4.-Crosses: $V$ vs. $B-V$ for extractions of $r<120$ pixels $\left(r<47^{\prime \prime}\right)$. Open circles: $r<53$ pixels $\left(r<21^{\prime \prime}\right)$ overlaid. The M5 mean locus is superposed.

1996) is superposed. This mean locus was obtained from data taken in the same observing run, and it is very similar to that of Sandquist et al. (1996). The CMD of a globular cluster is clearly seen for AL 3, with a BHB, a few giants, and a populated subgiant branch. The overall fit of the M5 mean locus indicates that $\mathrm{AL} 3$ has a similar metallicity of $[\mathrm{Fe} / \mathrm{H}]=-1.3 \pm 0.25$.

In Figure 5, we show the $V$ versus $B-V$ offset field at $4^{\prime}$ north corresponding to an extraction of $r<120$ pixels $\left(r<47^{\prime \prime}\right)$ and a more central one with $r<53$ pixels $\left(r<21^{\prime \prime}\right)$ overlaid. It shows two small area samplings dominated by the bulge population. A comparison of Figures 3 and 4 shows that the evolutionary sequences are very different. For $r<53$ pixels $(r<$ $21^{\prime \prime}$ ), we have 64 stars in the field, and 108 in the cluster area, showing about a $4 \sigma$ excess. In the $r<120$ pixels $\left(r<47^{\prime \prime}\right)$, we have 364 stars in the field, and 491 in the cluster area, showing about a $7 \sigma$ excess. We conclude that undoubtedly we are dealing with a star cluster. $V$ versus $V-I$ CMDs for the cluster and field indicate essentially the same features and conclusions.

In general, intermediate-metallicity bulge globular clusters that are relatively depleted in giants, with blue extended HBs, are related to a post-core-collapse structure (Fusi Pecci et al. 1993; Trager et al. 1995). Examples are NGC 6522 (Barbuy et al. 1994; Terndrup et al. 1998), NGC 6540 (Bica et al. 1994), HP 1 (Barbuy et al. 2006; Ortolani et al. 1997), and NGC 6558 (Rich et al. 1998). AL 3 appears to be one more such cluster.

Figure 6 shows $I$ versus $B-I$ for the same extractions as in Figure 4, where again the M5 mean locus is superposed. The blue straggler star (BSS) sequence of M5 is also shown,

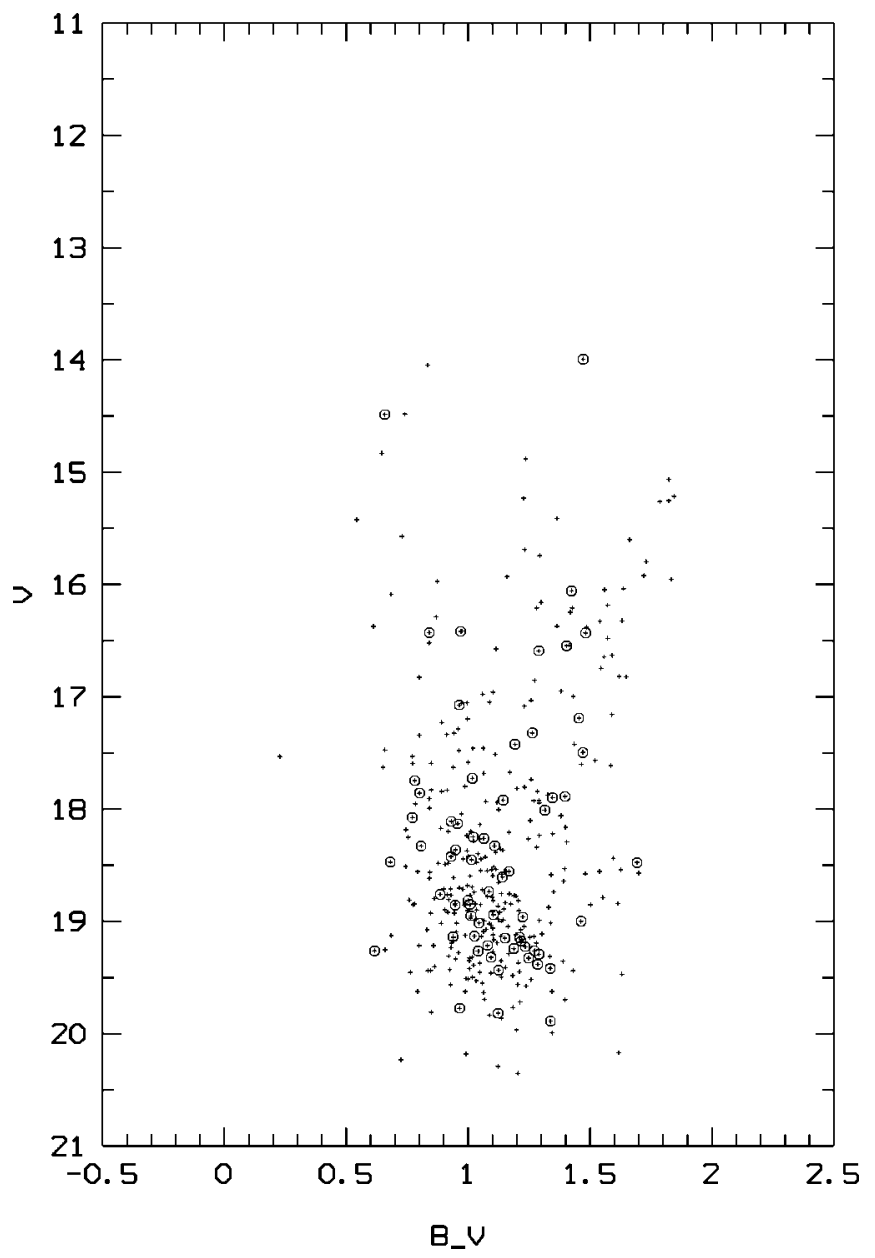

FIG. 5.-Same as Fig. 4, but for the offset field $4^{\prime}$ north.

indicating that some BSSs are present in AL 3. The good fit of the BSS locus between AL 3 and M5 suggests a comparable age for the clusters. Despite not having a clear TO locus, the locus of the BSS is a prolongation of the main sequence, providing information on the TO location.

\subsection{Reddening and Distance for AL 3}

The fit of M5 sequences to those of AL 3 in Figure 4 gives $V_{\mathrm{HB}}=15.7 \pm 0.2$ for the brightest part of the BHB. The color of the RGB at the HB level is $B-V=1.16 \pm 0.05$. The difference with respect to the fit with $\mathrm{M} 5$ is $\Delta(B-V)_{(\mathrm{AL} 3-\mathrm{M} 5)}=$ 0.33 , so that the reddening of $\mathrm{AL} 3$ is $E(B-V)=0.36$, considering $E(B-V)=0.03$ for M5 (Harris 1996). Adopting $R=3.1$, we obtain $A_{V}=1.12$, and using $M_{V}^{\mathrm{HB}}=0.7$ suitable for the cluster metallicity (Buonanno et al. 1989) results in $(m-M)_{0}=13.9$, and the distance to the Sun $d_{\odot}=6.0 \pm$ $0.5 \mathrm{kpc}$ for AL 3. Assuming the distance of the Sun to the Galaxy center to be $R_{\odot}=8.0 \mathrm{kpc}$ (Reid 1993), the galactocentric coordinates are $X=-2.0 \mathrm{kpc}(X>0$ is on the other side of the Galaxy), $Y=-0.35 \mathrm{kpc}$, and $Z=-0.55 \mathrm{kpc}$. The distance from the Galactic center is $R_{\mathrm{GC}}=2.1 \mathrm{kpc}$; thus, $\mathrm{AL} 3$ is an inner bulge cluster and should be incorporated into the sample of Barbuy et al. (1998), in terms of both the angular and the radius separation from the center. Bica et al. (2006) derived a distance of the Sun to the Galaxy center of $R_{\odot}=7.2 \mathrm{kpc}$, using globular clusters. In this case, $X=-2.0 \mathrm{kpc}, Y=-0.35 \mathrm{kpc}$, $Z=-0.55 \mathrm{kpc}, R_{\mathrm{GC}}=1.2 \mathrm{kpc}$, and $\mathrm{AL} 3$ is even closer to the center. 


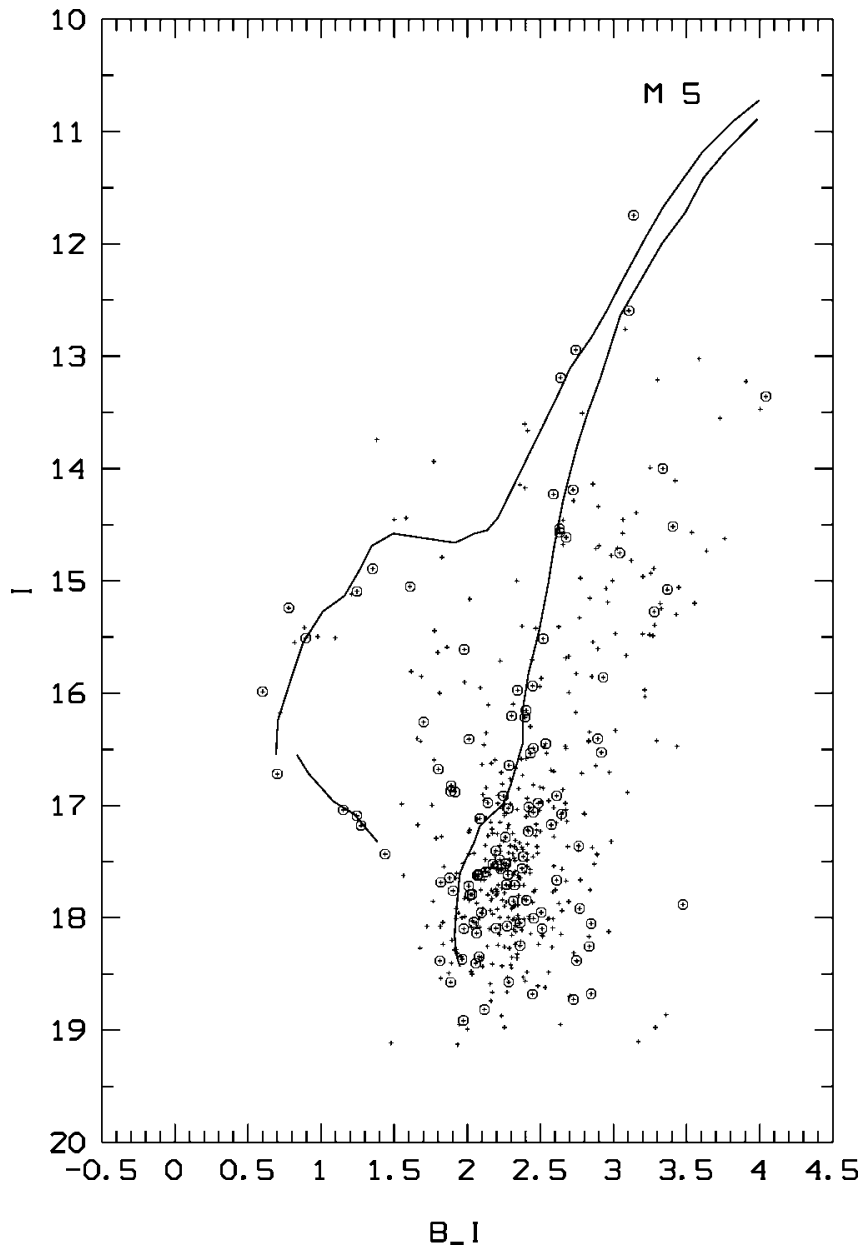

FIG. 6.-Same as Fig. 4, but for $I$ vs. $B-I$.

We estimated the cluster-observed integrated magnitude in the $60 \mathrm{~s} V$ image, by flux integration within $r<120^{\prime \prime}$ and subtraction of the background measured around the cluster in a factor 6 larger area. The sky background error is $\approx 5 \%$, while the average cluster flux is only $10 \%$ above the background.
We derived $V_{t}=11.0 \pm 0.4$, and with reddening and distance values as above we obtained $M_{V}=-4.0 \pm 0.45$ for $\mathrm{AL} 3$. Comparing to the Harris (1996) compilation, we conclude that AL 3 is among one of the most intrinsically faintest globular clusters, only somewhat more luminous than AM 4, Pal 1, E3, and Pal 13.

\section{CONCLUDING REMARKS}

We identify the object AL 3, discovered by Andrews \& Lindsay (1967), as a new globular cluster in the Galaxy. Since the discovery of Djorgovski 1, and the identification of ESO 456-SC38 and NGC 6540 (Djorgovski 1987), AL 3 is the next inner bulge globular cluster identified. We find a metallicity of $[\mathrm{Fe} / \mathrm{H}] \approx-1.3 \pm 0.25$. It is located in the inner bulge, at a distance $d_{\odot}=6 \pm 0.5 \mathrm{kpc}$. It appears to be another bulge intermediate-metallicity globular cluster, with a blue extended horizontal branch and a depleted red giant branch (Rich et al. 1998). AL 3 color-magnitude diagrams suggest that the cluster may have about 10 giants, similar to Palomar 13 (Siegel et al. 2001), and may be somewhat more massive than AM 4, which has no giants and a mass less than $1000 M_{\odot}$ (Inman \& Carney 1987). We are dealing with one of the least massive globular clusters, similar to several Palomar clusters. The contrast in metallicity between AL 3 and the bulge made possible for us to identify the cluster; a metal-rich globular cluster in the same condition would be indistinguishable from the bulge population. Other faint globular clusters may still be found in the bulge. AL 3, as a new intermediate-metallicity globular cluster located in the bulge, is another interesting target for abundance determinations and chemical enrichment studies of the Galaxy.

We acknowledge partial financial support from the Brazilian agencies $\mathrm{CNPq}$ and Fapesp, and from the Ministero dell'Università e della Ricerca Scientifica e Tecnologica (MURST) under the program on "Fasi iniziali di Evoluzione dell'Alone e del Bulge Galattico" (Italy).

\section{REFERENCES}

Andrews, A. D., \& Lindsay, E. M. 1967, Irish Astron. J., 8, 126

Barbuy, B., Bica, E., \& Ortolani, S. 1998, A\&A, 333, 117

Barbuy, B., Ortolani, S., \& Bica, E. 1994, A\&A, 285, 871

Barbuy, B., et al. 2006, A\&A, 449, 349

Bica, E., Bonatto, C., Barbuy, B., \& Ortolani, S. 2006, A\&A, 450, 105

Bica, E., Ortolani, S., \& Barbuy, B. 1994, A\&A, 283, 67

Buonanno, R., Corsi, C.E., \& Fusi Pecci, F. 1989, A\&A, 216, 80

Carraro, G. 2005, ApJ, 621, L61

Carraro, G., Janes, K. A., \& Eastman, J. D. 2005, MNRAS, 364, 179

Da Costa, G. S. 1995, PASP, 107, 937

Diego, F. 1985, PASP, 97, 1209

Djorgovski, S. 1987, ApJ, 317, L13

Fusi Pecci, F., Ferraro, F. R., Bellazzini, M., Djorgovski, S., Piotto, G., \& Buonanno, R. 1993, AJ, 105, 1145

Harris, W. E. 1996, AJ, 112, 1487

Harris, W. E., Phelps, R. L., Madore, B. F., Pevunova, O., \& Skiff, B. A. 1997, AJ, 113, 688

Hurt, R. L., Jarrett, T. H., Kirkpatrick, J. D., Cutri, R. M., Schneider, S. E., Skrutskie, M., \& van Driel, W. 2000, AJ, 120, 1876

Inman, R. T., \& Carney, B. W. 1987, AJ, 93, 1166

Irwin, M. J., Demers, S., \& Kunkel, W. E. 1995, ApJ, 453, L21

Kobulnicky, H. A., et al. 2005, AJ, 129, 239

Landolt, A. U. 1983, AJ, 88, 439
Landolt, A. U. 1992, AJ, 104, 340

Lauberts, A. 1982, ESO/Uppsala Survey of ESO(B) Atlas (Garching: ESO)

Ortolani, S., Barbuy, B., \& Bica, E. 1990, A\&A, 236, 362

Ortolani, S., Bica, E., \& Barbuy, B. 1993, A\&A, 273, 415

- 1997, MNRAS, 284, 692 2000, A\&A, 361, L57

Reid, M. 1993, ARA\&A, 31, 345

Rich, R. M., Ortolani, S., Bica, E., \& Barbuy, B. 1998, AJ, 116, 1295

Sandquist, E., Bolte, M., Stetson, P. B., \& Hesser, J. E. 1996, ApJ, 470, 910

Sarajedini, A., \& Geisler, D. 1996, AJ, 112, 2013

Siegel, M. H., Majewski, S. R., Cudworth, K. M., \& Takamiya, M. 2001, AJ, 121,935

Stetson, P. B. 1987, PASP, 99, 191

Tavarez, M., \& Friel, E. D. 1995, AJ, 110, 223

Terndrup, D. M. 1988, AJ, 96, 884

Terndrup, D. M., Popowski, P., Gould, A., Rich, R. M., \& Sadler, E. M. 1998, AJ, 115, 1476

Trager, S., King, I. R., \& Djorgovski, S. 1995, AJ, 109, 218

van den Bergh, S., \& Hagen, G. L. 1975, AJ, 80, 11

Weinberger, R. 1995, PASP, 107, 58

Whiting, A. B., Hau, G. K. T., \& Irwin, M. 2002, ApJS, 141, 123

Willman, B., et al. 2005, AJ, 129, 2692 